Randomized Trial

\title{
Serious Adverse Events Associated with Readmission Through One Year After Vertebral Augmentation with Either a Polyetheretherketone Implant or Balloon Kyphoplasty Follow-up Analysis of the KAST Randomized Controlled Trial Comparing the Kiva Vertebral Compression Fracture Treatment System
}

Douglas P. Beall, MD1, Jeffrey D. Coe, MD², Mark Mcllduff, MBA33, Daniel Bloch, PhD4, John Hornberger, MD, Christopher Warner, MD', and Sean M. Tutton, MD ${ }^{6}$

From: ${ }^{2}$ Clinical Radiology of Oklahoma, Edmond, OK; ${ }^{2}$ Silicon Valley Spine Institute, Campbell, CA; 'Boston Biomedical Associates, Marlborough, MA; 4Professor Emeritus, Biostatistics, Stanford University Medical School, Stanford, CA; ${ }^{5}$ Cedar Associates and Stanford University, Menlo Park, CA; ${ }^{6}$ Froedtert Memorial Lutheran Hospital and Medical College of Wisconsin Milwaukee,

WI

Address

Correspondence: Douglas P. Beall, MD

Clinical Radiology of Oklahoma 610 NW 14th St Oklahoma City, OK

E-mail: db@clinrad.org

Disclaimer: There was no external funding in the preparation of this manuscript.

Conflict of interest: See

pg. 527.

Manuscript received: 01-03-2017

Revised manuscript received: 03-20-2017

Accepted for publication: 03-30-2017

Free full manuscript: www. painphysicianjournal. com
Background: The KAST (Kiva Safety and Efficacy) investigation device exempt (IDE) study indicated that the majority of patients responded equally well to vertebral augmentation using either an implantbased approach or balloon kyphoplasty (BK). Additional investigation has suggested that a subset of patients may benefit further by avoiding repeated readmissions due to serious adverse events (SAEs) if they receive one vertebral augmentation approach over another.

Objectives: The primary aim was to assess the effect of 2 different augmentation procedures on readmission rates for SAEs.

Study Design: The KAST trial is a pivotal, multicenter, randomized, controlled trial conducted to evaluate an implant-based vertebral augmentation approach (implant) against BK. Post-hoc analysis was performed to evaluate SAEs and readmission rates.

Setting: Twenty-one sites in North America and Europe.

Methods: The treatment effect of vertebral implant versus BK on SAEs requiring unplanned readmission was evaluated by estimating the risk of SAEs associated with readmissions in KAST while controlling for key baseline covariates using multivariate Poisson regression modeling.

Results: Forty (27.8\%) patients with implants had 69 SAEs associated with readmission compared to 44 (31.2\%) patients with BK having 103 events. The risk for all SAEs leading to readmission was 34.4\% lower with the implant than for BK $(95 \%$ confidence interval $=11.1 \%, 51.7 \% ; P<0.01)$. Multivariate analysis showed that the risk of SAEs associated with readmission was decreased in subjects treated with the implant compared to BK, and increased in patients with prior histories of vertebral compression fractures (VCFs) or significant osteoporosis.

Limitations: The power of the KIVA study was based on clinical efficacy criteria to meet FDA requirements and recommendations for equivalency or noninferiority. The primary endpoint in this posthoc analysis is SAEs associated with readmissions; as a result, the sample size is underpowered, although the results remain significant.

Conclusion: The augmentation approaches compared here have similar pain relief and quality of life effects; the implant showed a lower risk of readmissions.

Trial Registration: ClinicalTrials.gov Identifier: NCT01123512

Key words: Vertebral compression fracture, kiva implant, balloon kyphoplasty, vertebroplasty, health economics, osteoporosis

Pain Physician 2017; 20:521-528 
$\mathbf{V}$ ertebral compression fractures (VCFs) cause significant pain and disability. They are often seen concomitantly with cardiovascular and pulmonary disorders, along with other types of fractures. Patients with VCFs are initially managed nonsurgically, but when symptoms are sufficiently severe and persistent, or there is a progressive collapse, a vertebral augmentation procedure is an appropriate option for consideration (1).

The primary goals of vertebral augmentation are pain relief and improved mobility and quality of life (2-6). Vertebral augmentation shows the potential to reduce the risk of early mortality and incidence of morbidity events over nonsurgical management (NSM) (7-9). In a longitudinal study sample consisting of over one million Medicare patients who received treatment for a VCF, those who underwent vertebral augmentation had a significantly lower risk of myocardial infarction/cardiac complications, deep vein thrombosis (DVT), pneumonia, and urinary tract infection (UTI) during the year after treatment was initiated, than those who were managed nonsurgically (7).

With balloon kyphoplasty (BK), image guidance is used to direct inflatable bone tamps (Fig. 1A) into the fractured vertebral body to create a cavity for injection of bone cement. This approach allows for improved placement of bone cement compared to vertebroplasty alone. Edidin and colleagues (7) reported that patients who had BK tended to have a significantly less risk of having a subsequent augmentation or repair for compression fracture, and had a significantly reduced risk for having post-procedure pneumonia, pulmonary embolism, or UTI, than those who were treated with vertebroplasty. Gu et al (5) reported that BK was associated with significantly lower odds of developing new fractures than vertebroplasty.

The kiva safety and effectiveness trial (KAST) was a multicenter, randomized investigational device exempt (IDE) trial conducted to demonstrate noninferiority of an implant-based vertebral augmentation system to BK for key clinical outcomes, including pain reduction, functional improvement, and device-related complications (10). The implant used in the study was an expandable coil polyether ether ketone (PEEK) device (Fig. 1B) that is placed by using a transpedicular approach into the vertebral body, followed by an injection of a small quantity of cement through the implant into the treated area. The implant is designed to improve containment of the injected cement, enhance structural support and sagittal alignment, and require smaller amounts of cement than BK.

Previous investigations have suggested evidence of potentially reduced subsequent adjacent fractures with the implant compared to BK (11-13). On the basis of these previous reports, we hypothesized that KAST patients having vertebral augmentation with the implant would demonstrate evidence of reduced morbidity compared to patients assigned to BK. The purpose of this study was to evaluate the serious adverse events (SAEs) associated with readmission through one year after treatment in patients randomly assigned to receive vertebral augmentation using either the implant or BK.

\section{Methods}

The KAST study design and methods have been described previously (10). In summary, 21 sites in North America and Europe enrolled a total of 300 patients. The 'as-treated' (AT) analysis population in KAST was used for this study. Ninety-five percent (285/300) of KAST patients met criteria for the AT population, which included all randomized patients in whom the intended procedure was performed and a technically successful result was obtained. There were 144 patients in the implant group and 141 in the BK group. A total of 235/285 patients $(88.8 \% ; 127$ implant and $126 \mathrm{BK})$ completed the final study visit at 12 months.

In KAST, an adverse event or suspected adverse event was considered an SAE if it satisfied at least one of the following criteria: (1) resulted in death, lifethreatening illness or injury, (2) required inpatient hospitalization or prolonged existing hospitalization, (3) resulted in permanent impairment of a body structure or body function, or (4) resulted in a medical or surgical intervention to prevent permanent impairment to a body structure or function. An independent physician adjudicator (IPA) reviewed all of the site-reported SAEs; adjudicated records were maintained as the regulatory dataset and used for the IDE study FDA submission. For this study, the SAEs seen in KAST were categorized in accordance with the Medical Dictionary for Regulatory Activities (MedDRA) coding system into "System Organ Class" (SOC) and "Preferred Term" (PT) subgroups. The number of SAEs associated with readmissions was tabulated for each patient.

A t-test was used to test the difference between 2 means. A chi-square test was used to test the difference between 2 proportions in cases where the number of events was greater than 10, and the Fisher's exact test used to test the difference between 2 proportions in 


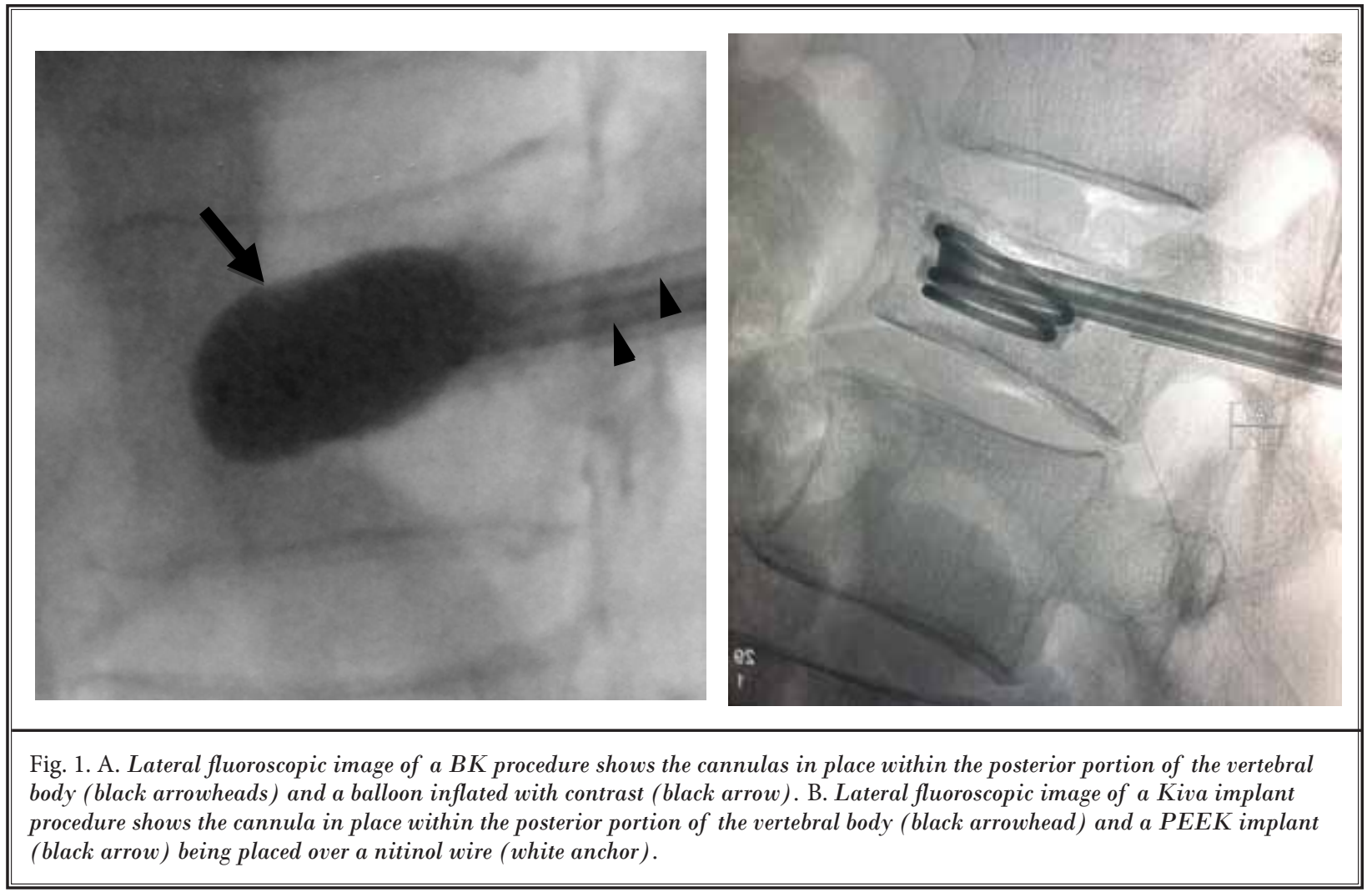

cases where the number of events were less than 10. The Mann-Whitney test (same as the Wilcoxon 2-sample rank test) was used to test the difference between the 2 treatment groups for ordered categorical data. Statistical significance was accepted at $P<0.05$.

Poisson regression models were generated to examine the treatment effect on SAEs requiring an unplanned readmission. The key independent variables tested were treatment group (implant or BK) and patient follow-up duration (months). The dependent variable for each model was the log-transformed Poisson distribution of the count of SAEs requiring an unplanned readmission. Additional univariate Poisson models were generated to test the effect of key baseline covariates on the same outcome. Relevant subject level baseline covariates had been pre-specified in the KAST Study Statistical Analysis Plan and were evaluated for significance in predicting SAEs associated with readmission. The baseline covariates that were evaluated included age, gender, body mass index, smoking history, prior history of VCF, narcotic use, visual analogue score (VAS) score for back pain and Oswestry Disability Index scores, duration of symptoms, duration of conservative treatment, and DEXA spine T-score. Statistical analyses were performed using SAS Version 9.4 Software (SAS Institute, Inc., Cary, NC).

\section{Results}

The study groups had comparable demographic information at baseline for most characteristics (Table 1). The implant group had a significantly higher proportion of former smokers and patients with a history of thoracolumbar junction fractures, while the BK group had a significantly higher proportion of patients who reported having a nervous system disorder such as urinary incontinence, fibromyalgia, or radiculopathy not related to the VCF. Having a history of osteoporotic VCF at the time of enrollment was increased toward the implant group with borderline significance $(P=0.079)$.

During the one-year follow-up, $27.8 \%$ implant patients and $31.2 \%$ BK patients had at least one SAE associated with readmission (Table 2). The implant group had a total of 69 SAEs associated with readmission compared to the BK group who had a total of 103 events. In every SOC category other than 'Infections,' the BK group had a higher number of SAEs associated with readmission. The difference between treatment groups for risk of infection was not statistically signifi- 
Table 1. Baseline Characteristics in KAST Patients.

\begin{tabular}{|c|c|c|c|}
\hline Characteristic & $\begin{array}{c}\text { Implant } \\
(\mathrm{n}=144)\end{array}$ & $\begin{array}{c}\text { BK } \\
(n=141)\end{array}$ & $P$-value \\
\hline Age (yrs) & $76( \pm 9)$ & $75( \pm 10)$ & 0.375 \\
\hline Female & $105(73 \%)$ & $106(75 \%)$ & 0.663 \\
\hline \multicolumn{4}{|l|}{ Smoking History } \\
\hline Non-smoker & $64(44 \%)$ & $78(55 \%)$ & $0.066 \ddagger$ \\
\hline Former smoker & $60(42 \%)$ & $42(30 \%)$ & $0.036 \dagger$ \\
\hline Current smoker & $20(14 \%)$ & $21(15 \%)$ & 0.809 \\
\hline Prior Spinal Surgeries & $35(24 \%)$ & $27(19 \%)$ & 0.291 \\
\hline Single-level procedures & $19(13 \%)$ & $15(11 \%)$ & 0.506 \\
\hline Multi-level procedures & $17(12 \%)$ & $15(11 \%)$ & 0.755 \\
\hline History of Osteoporotic VCF & $70(49 \%)$ & $54(38 \%)$ & $0.079 \ddagger$ \\
\hline Thoracic (levels T1-T10) & $29(20 \%)$ & $29(21 \%)$ & 0.928 \\
\hline Thoracolumbar junction (levels T11, T12, L1) & $42(29 \%)$ & $27(19 \%)$ & $0.048 \dagger$ \\
\hline Lumbar (levels L2-L5) & $32(22 \%)$ & $27(19 \%)$ & 0.522 \\
\hline \multicolumn{4}{|l|}{ Significant Medical Conditions, by SOC } \\
\hline Cardiovascular disorders & $110(69.4 \%)$ & $100(70.1 \%)$ & 0.295 \\
\hline Respiratory, thoracic, \& mediastinal disorders & $28(19.4 \%)$ & $38(27.0 \%)$ & 0.133 \\
\hline Nervous system disorders & $38(26.4 \%)$ & $54(38.4 \%)$ & $0.032 \dagger$ \\
\hline None reported & $7(5 \%)$ & $6(4 \%)$ & 1.00 \\
\hline
\end{tabular}

Values are mean \pm standard deviation or $\mathrm{N}(\%)$

$\dagger$ Denotes a significant term at $P$-value $<0.05$.

$\ddagger$ Denotes a significant term at $P$-value $<0.10$.

cant. A significantly higher proportion of BK patients had a cardiovascular disorder SAE during the one-year follow-up ( $8.5 \%$ versus $2.8 \% ; P<0.041$ ). Having a diagnosis of pneumonia at the time of death was of borderline significance higher in the BK group $(2.8 \%$ versus $0 \% ; P<0.059)$.

Univariate modeling with treatment group as the independent variable showed that the risk of having an SAE associated with readmission was significantly decreased in the implant group compared to the BK group (risk estimate $=0.656[0.483-0.889], P=0.007$; Table 3 ). This risk estimate is equivalent to patients who were treated with the implant in KAST having an estimated $34.4 \%$ decreased risk of having an SAE associated with readmission over the BK group. A lower risk of having a cardiac disorder during the one-year follow-up with the implant compared to BK was of borderline significance $(P<0.053)$.

The multivariate regression model is summarized in Table 4. Besides treatment group, previous history of VCF and more profound osteoporosis (DEXA baseline spine T-score of -2.5 or more severe) were seen as significant predictors for SAEs associated with unplanned readmission. This model suggests that the risk of SAEs associated with unplanned readmission was increased by $38.2 \%$ in patients treated with BK (compared to implant), $42.9 \%$ in patients with a previous history of VCFs, and $79.6 \%$ in patients with more severe osteoporosis. Conversely, treatment with the implant, no prior history of VCF, and less severe osteoporosis before treatment significantly decreased the risk of SAEs associated with readmission during the first year after the index vertebral augmentation treatment.

\section{Discussion}

The risk of readmissions due to SAEs was significantly decreased by $34 \%$ with the implant compared to BK. Multivariate analysis also showed the importance of prior history of VCF and more severe osteoporosis on the risk of readmissions

In a previous, non-randomized study consisting of over one million Medicare beneficiaries, Edidin et al (7) found that vertebral augmentation provided substantial benefits over NSM during the one year after an index treatment was initiated. Vertebral augmentation performed using BK was associated with a $55 \%$ reduced risk of early mortality through one year after treatment, along with a significantly reduced risk of specific morbidities. Compared to BK patients, NSM patients were more likely to be readmitted with pneumonia $(O R=1.19)$, die with a pneumonia diagnosis (OR $=1.41$ ), or have a myocardial infarction or cardiac complications (OR = 1.12), UTI (OR = 1.14), or DVT $(O R=1.12)$. In this study, we found that BK patients were more likely than implant patients to have any SAE associated with readmission, and had a borderline significant higher risk of having a cardiac or vascular event.

The per-patient readmissions rate that may be anticipated in patients prior to receiving treatment for an index VCF has been estimated to be approximately $35 \%$ (8). For NSM patients participating in the FREE study, a randomized, controlled trial comparing $\mathrm{BK}$ to NSM, Wardlaw and colleagues 
Serious Adverse Events Associated with Re-Admissions after Vertebral Augmentation

Table 2. SAEs associated with readmission and mortality through one year in KAST patients.

\begin{tabular}{|c|c|c|c|c|c|}
\hline \multirow{2}{*}{ Serious Adverse Events } & \multicolumn{2}{|c|}{$\mathbf{N}(\%)$} & \multirow{2}{*}{$P$-value* } & \multicolumn{2}{|c|}{ \# Events } \\
\hline & Implant & BK & & Implant & BK \\
\hline SAEs associated with readmission & $\mathrm{N}=144$ & $\mathrm{~N}=141$ & & & \\
\hline 30-day & $12(8.3 \%)$ & $15(10.6 \%)$ & 0.506 & 14 & 19 \\
\hline 90-day & $18(12.5 \%)$ & $22(15.6 \%)$ & 0.451 & 26 & 29 \\
\hline 1-year & $40(27.8 \%)$ & $44(31.2 \%)$ & 0.526 & 69 & 103 \\
\hline \multicolumn{6}{|l|}{ Number of unplanned readmissions in 1-year } \\
\hline 0 & $104(72.2 \%)$ & $97(68.8 \%)$ & 0.385 & 0 & 0 \\
\hline 1 & $24(16.7 \%)$ & $22(15.6 \%)$ & & 24 & 22 \\
\hline 2 & $11(7.6 \%)$ & $9(6.4 \%)$ & & 22 & 19 \\
\hline $3+$ & $5(3.5 \%)$ & $13(9.2 \%)$ & & 23 & 62 \\
\hline \multicolumn{6}{|l|}{ Reasons for Unplanned Readmission } \\
\hline Injury and procedural complications & $13(9.0 \%)$ & $17(12.1 \%)$ & 0.405 & 19 & 29 \\
\hline Fracture, spinal compression & $8(5.6 \%)$ & $12(8.5 \%)$ & & 11 & 19 \\
\hline Fracture, other & $4(2.8 \%)$ & $3(2.1 \%)$ & & 6 & 4 \\
\hline Fall & $2(1.4 \%)$ & $3(2.1 \%)$ & & 2 & 5 \\
\hline Airway complication of anesthesia & 0 & $1(0.7 \%)$ & & 0 & 1 \\
\hline Cardiovascular disorders & $4(2.8 \%)$ & $12(8.5 \%)$ & $0.041 \dagger$ & 4 & 20 \\
\hline Myocardial infarction or cardiac arrest & $1(0.7 \%)$ & $4(2.8 \%)$ & & 1 & 4 \\
\hline Cardiac failure congestive & $1(0.7 \%)$ & $2(1.4 \%)$ & & 1 & 4 \\
\hline Arrhythmia or atrial fibrillation & $2(1.4 \%)$ & $1(0.7 \%)$ & & 2 & 1 \\
\hline Acute coronary syn., unstable angina, dizziness & 0 & $2(1.4 \%)$ & & 0 & 3 \\
\hline Hypertension or hypotension & 0 & $3(2.1 \%)$ & & 0 & 3 \\
\hline Aortic stenosis, aortic aneurysm & 0 & $2(1.4 \%)$ & & 0 & 2 \\
\hline DVT & 0 & $1(0.7 \%)$ & & 0 & 1 \\
\hline Intermittent claudication & 0 & $1(0.7 \%)$ & & 0 & 1 \\
\hline Peripheral vascular disorder & 0 & $1(0.7 \%)$ & & 0 & 1 \\
\hline Respiratory, thoracic, and mediastinal disorders & $4(2.8 \%)$ & $6(4.3 \%)$ & 0.538 & 4 & 6 \\
\hline COPD & $2(1.4 \%)$ & $2(1.4 \%)$ & & 2 & 2 \\
\hline Pulmonary oedema & $2(1.4 \%)$ & $1(0.7 \%)$ & & 2 & 1 \\
\hline Pneumonia aspiration & 0 & $1(0.7 \%)$ & & 0 & 1 \\
\hline Pulmonary embolism & 0 & $1(0.7 \%)$ & & 0 & 1 \\
\hline Respiratory failure & 0 & $1(0.7 \%)$ & & 0 & 1 \\
\hline Nervous system disorders & 0 & $3(2.1 \%)$ & 0.120 & 0 & 4 \\
\hline Myasthenia gravis, presyncope, syncope & 0 & $3(2.1 \%)$ & & 0 & 3 \\
\hline Ischaemic stroke & 0 & $1(0.7 \%)$ & & 0 & 1 \\
\hline Infections & $12(8.3 \%)$ & $10(7.1 \%)$ & 0.695 & 17 & 10 \\
\hline Pneumonia & $5(3.5 \%)$ & $2(1.4 \%)$ & & 5 & 2 \\
\hline Urinary tract infection & $2(1.4 \%)$ & $4(2.8 \%)$ & & 2 & 4 \\
\hline Infection, other & $6(4.2 \%)$ & $4(2.8 \%)$ & & 10 & 4 \\
\hline Other SOC disorders & $20(13.9 \%)$ & $22(15.6 \%)$ & 0.683 & 25 & 34 \\
\hline \multicolumn{6}{|l|}{ Death } \\
\hline 30-day & $1(0.7 \%)$ & $1(0.7 \%)$ & 1.00 & & \\
\hline 1-year & $10(6.9 \%)$ & $9(6.4 \%)$ & 0.849 & & \\
\hline Death with pneumonia diagnosis & $0(0.0 \%)$ & $4(2.8 \%)$ & $0.059 \ddagger$ & & \\
\hline
\end{tabular}

$\dagger$ Denotes a significant term at $P$-value $<0.05$. $\ddagger$ Denotes a significant term at $P$-value $<0.10$. 
Pain Physician: September/October 2017: 20:521-528

Table 3. Univariate Poisson regression models for associations between treatment group and SAEs associated with unplanned readmissions by SOC category, accounting for by-patient follow-up duration.

\begin{tabular}{|c|c|c|c|c|c|}
\hline \multirow[b]{2}{*}{ MedDRA* SOC Category* Preferred Term } & \multicolumn{2}{|c|}{ Number of Events } & \multirow{2}{*}{$\begin{array}{c}\text { Risk } \\
\text { Estimate }\end{array}$} & \multirow[b]{2}{*}{$95 \%$ CI } & \multirow[b]{2}{*}{$P$-value } \\
\hline & $\begin{array}{l}\text { Implant } \\
(\mathrm{N}=144)\end{array}$ & $\begin{array}{c}\text { BK } \\
(\mathrm{N}=\mathbf{1 4 1})\end{array}$ & & & \\
\hline Injury and procedural complications & 19 & 29 & 0.641 & $0.360-1.144$ & 0.132 \\
\hline Spinal compression fracture & 11 & 19 & 0.567 & $0.270-1.191$ & 0.134 \\
\hline Infections & 17 & 10 & 1.669 & $0.764-3.646$ & 0.199 \\
\hline Cardiac disorders & 4 & 12 & 0.327 & $0.106-1.015$ & $0.053 \ddagger$ \\
\hline Respiratory, thoracic, and mediastinal disorders & 4 & 6 & 0.653 & $0.184-2.315$ & 0.509 \\
\hline Vascular disorders & 0 & 8 & $\$$ & $\$$ & $\$$ \\
\hline Nervous system disorders & 0 & 4 & $\$$ & $\$$ & $\$$ \\
\hline Other SOC categories & 25 & 34 & 0.722 & $0.431-1.210$ & 0.217 \\
\hline $\begin{array}{l}\text { Total Count of SAEs Associated wtih Unplanned } \\
\text { Readmission }\end{array}$ & 69 & 103 & 0.656 & $0.483-0.889$ & $0.007 \dagger$ \\
\hline
\end{tabular}

$\dagger$ Denotes a significant term at $P$-value $<0.05$. $\ddagger$ Denotes a significant term at $P$-value $<0.10$. $\S$ Regression model is non-converged with the low counts; risk estimates and $P$-values are not available.

(14) reported a rate of $36 \%$. The readmissions rate in KAST for both the implant and BK groups were slightly lower than $35 \%$, with $28 \%$ and $31 \%$ of implant and BK patients, respectively, having at least one SAE associated with readmission during the one-year follow-up. McCullough et al (8) also reported that approximately $20 \%$ of patients had 2 or more inpatient readmissions and $10 \%$ had 3 or more readmissions. The implant and BK patients in KAST demonstrated roughly similar proportions, with $24 \%$ and $22 \%$, respectively, having 2 or more readmissions and $4 \%$ and $9 \%$, respectively, having 3 or more readmissions. The results of these studies suggest that approximately one in 3 VCF patients may be anticipated to be readmitted at least once during the year after an index vertebral augmentation procedure, with one in 10 having repeated readmissions during that same timeframe. Implant-based vertebral augmentation, however, may significantly reduce the risk for repeated readmissions over BK.

There may be several reasons for the observed significant difference in readmissions between the treatment groups in this study. There is a possibility that spinal sagittal balance may be positively impacted by the implant, which is supported by the significantly enhanced kyphotic angle measure seen in implant patients over BK reported by Korovessis and colleagues (12). In KAST, post-operative kyphotic angle measures did not differ significantly between treatment arms, although a positive trend was noted in favor of the implant group (patients with improvement or maintenance: $75.6 \%$ versus $65.6 \%$; Bayesian confidence interval $=-1.13 \%$, $20.69 \%)$. There is also evidence that the implant may be associated with reduced subsequent fractures than BK (11-13). Improved kyphotic angle has a positive correlation with improved vital capacity (15), which may affect the overall well-being of a patient. The study of the potential restoration of kyphotic angle and spinal sagittal balance with treatment of VCFs, in conjunction with reduced subsequent readmissions, may be an area that is worthy of additional research.

One of the aims for improving cement placement with vertebral augmentation is avoidance of extravasation and the risks it poses for SAEs. An ex vivo biomechanical study showed that vertebral augmentation with the implant exhibited similar biomechanical performance to BK (16), but that risk of extravasation may be reduced due to the containment mechanism of the implant design and the smaller amount of cement volume that may be required for the procedure (17). In KAST, treating physicians reported using significantly less cement with Kiva than BK $(2.37 \pm 1.06 \mathrm{~mL}$ versus $5.38 \pm 2.17 \mathrm{~mL}$, respectively). It is not known if the relative reduction in the amount of cement used with the implant compared to BK is clinically relevant, but this too might also warrant further consideration.

An important limitation of this secondary analysis of the KAST trial concerns the use of SAEs associated with readmissions as the main endpoint. SAEs are routinely collected in clinical trials and must be reported to the FDA. Readmission rates, on the other hand, are less commonly a required reported endpoint; most trials are not powered to detect a difference in this endpoint, and perhaps this should change as a matter of policy because payments (e.g., CMS) are being tied to the 
Serious Adverse Events Associated with Re-Admissions after Vertebral Augmentation

Table 4. Final multivariate Poisson regression model predicting count of SAEs associated with unplanned readmissions.

\begin{tabular}{|l|c|c|c|c|c||}
\hline \multicolumn{1}{|c|}{ Variable } & Label & $\begin{array}{c}\text { Log-transformed } \\
\text { Estimate [1] }\end{array}$ & $\begin{array}{c}\text { Risk } \\
\text { Estimate }\end{array}$ & 95\% CI & $\boldsymbol{P}$-value \\
\hline Intercept & & -0.911 & & & \\
\hline \multirow{2}{*}{ Treatment Group } & Kiva & -0.323 & 0.724 & $0.524-0.999$ & 0.049 \\
\hline \multirow{2}{*}{\begin{tabular}{l} 
Prior History of VCF \\
\cline { 2 - 6 }
\end{tabular}} & BK & - & 1.382 & $1.001-1.909$ & - \\
\hline $\begin{array}{l}\text { Profound Osteoporosis (DEXA Spine } \\
\text { T-score at Baseline) }\end{array}$ & Yes & 0.357 & 1.429 & $1.031-1.982$ & 0.032 \\
\cline { 2 - 6 } & -2.5 or worse & - & 0.700 & $0.505-0.970$ & - \\
\hline
\end{tabular}

[1] Final Model: count $=\mathrm{e}^{(-0.911-0.323 * \text { Kiva }+0.357 * \mathrm{VCF}+0.585 * \text { Osteoporotic })}$

avoidance of readmissions. The power of the study was based on clinical efficacy criteria to meet FDA requirements and recommendations. Although underpowered to do so, it seems essential to report clinically meaningful higher rates of SAEs associated with readmissions for BK versus implants, especially as this was found in a randomized-controlled trial.

Further investigation of implant-based vertebral augmentation compared to BK is important to validate this study's findings. We observed a significantly greater risk for SAEs associated with readmissions in patients with more profound osteoporosis and with prior VCFs, but patients appeared to benefit from fewer readmissions with one vertebral augmentation approach compared to the other. In a study such as the one conducted here, revisiting previous investigational sites to obtain additional data after closing the study is a difficult, if not impossible, task to undertake. In the future, it may be beneficial for study sponsors and investigators conducting randomized, controlled trials to collect more complex SAE data prospectively as a means to further enrich the depth of knowledge regarding healthcare options.

The KAST data indicated that the majority of patients responded equally well to either vertebral augmentation approach, but there may be a subset of patients who may benefit by avoiding repeated readmissions if they receive one treatment approach over another. The subset of patients with prior VCFs and those with more profound osteoporosis appear to be at an especially high risk for readmissions and may be a vulnerable population to study further. It is interesting to note that the implant group had a higher percentage of subjects with a history of VCF $(P=0.079)$ but still yielded the observation of a reduced rate of SAEs associated with readmissions over the BK control group.

Potential reduction in readmissions and downstream morbidity and their intending cost savings to the healthcare system are worthy of additional investigation. This will be compounded as the population ages and an increasing number of individuals are at risk of VCFs. Employers and public and commercial payers are striving to manage the challenges of healthcare for this population with incentives that align the clinical needs of the patients with provider organizations. These findings warrant further study of the most common reasons for readmissions to help providers target subgroups at higher risk.

\section{Conflicts of Interest and Source of Funding}

Dr. Beall is a consultant for Benvenue Medical Inc.; Dr. Coe receives research support and is a consultant for Benvenue Medical Inc.; Mr. Mcllduff is a consultant for Benvenue Medical Inc.; Dr. Bloch is a consultant for Benvenue Medical Inc; Dr. Hornberger consulted for Benevue Medical Inc., but is no longer a consultant; Dr. Tutton was the principal investigator of KAST and is a consultant for Benvenue Medical Inc. 


\section{References}

1. Goldstein CL, Chutkan NB, Choma TJ, Orr RD. Management of the elderly with vertebral compression fractures. Neurosurgery 2015; 77:S33-S45.

2. Anderson PA, Froyshteter AB, Tontz WL Jr. Meta-analysis of vertebral augmentation compared with conservative treatment for osteoporotic spinal fractures. J Bone Min Res 2013; 28:372-382.

3. Chen LX, Li YL, Ning GZ, Li Y, Wu QL, Guo JX, Shi HY, Wang XB, Zhou Y, Feng SQ. Comparative efficacy and tolerability of three treatments in old people with osteoporotic vertebral compression fracture: A network meta-analysis and systematic review. PLOS ONE 2015; 10:eo123153.

4. Papanastassiou ID, Phillips FM, Van Meirhaeghe J, Berenson JR, Andersson GB, Chung G, Small BJ, Aghayev K, Vrionis FD. Comparing effects of kyphoplasty, vertebroplasty, and non-surgical management in a systematic review of randomized and non-randomized controlled studies. Eur Spine J 2012; 21:1826-1843.

5. Gu CN, Brinjikji W, Evans AJ, Murad $\mathrm{MH}$, Kallmes DF. Outcomes of vertebroplasty compared with kyphoplasty: A systematic review and meta-analysis. J Neuro Intervent Surg 2015; 0:1-7.

6. Guo JB, Zhu Y, Chen BL, Xie B, Zhang WY, Yang YJ, Yue YS, Wang XQ. Surgical versus non-surgical treatment for vertebral compression fracture with osteopenia: A systematic review and metaanalysis. PLoS ONE 2015; 10:e0127145.

7. Edidin AA, Ong KL, Lau E, Kurtz SM. Morbidity and mortality after vertebra fractures. Spine 2015; 40:1228-1241.

8. McCullough BJ, Comstock BA, Deyo RA, Kreuter W, Jarvik JG. Major medical outcomes with spinal augmentation versus conservative therapy. JAMA Intern Med 2013; 173:1514-1521.

9. Chen AT, Cohen DB, Skolasky RL. Impact of nonoperative treatment, vertebroplasty, and kyphoplasty on survival and morbidity after vertebral compression fracture in the Medicare population. J Bone Joint Surg Am 2013; 95:1729-1736.

10. Tutton SM, Pflugmacher R, Davidian M, Beall DP, Facchini FR, Garfin SR. KAST Study: The Kiva System as a vertebra augmentation treatment: A safety and effectiveness trial. Spine 2015; 40:865-875.

11. Otten LA, Bornemann R, Jansen TR, Kabir K, Pennekamp PH, Wirtz DC, Stuwe $\mathrm{B}$, Pflugmacher R. Comparison of balloon kyphoplasty with the new Kiva VCF System for the treatment of vertebral compression fractures. Pain Physician 2013; 16: $\mathrm{E}_{505}-\mathrm{E}_{512}$

12. Korovessis P, Vardakastanis K, Repantis T, Vitsas V. Balloon kyphoplasty versus KIVA vertebral augmentation - comparison of 2 techniques for osteoporotic vertebral body fractures. Spine 2013; 38:292-299.

13. Beall DP, Olan WJ, Kakad P, Li Q, Hornberger J. Economic analysis of Kiva VCF treatment system compared to balloon kyphoplasty using randomized Kiva safety and effectiveness trial (KAST) data. Pain Physician 2015; 18:E299-E306.

14. Wardlaw D, Cummings SR, Van Meirhaeghe J, Bastian L, Tillman JB, Ranstam J, Eastell R, Shabe P, Talmadge K, Boonen S. Efficacy and safety of balloon kyphoplsty compared with nonsurgical care for vertebral compression fracture (FREE): A randomized controlled trial. Lancet 2009; 373:1016-1024.

15. Dong RB, Chen L, Gu Y, Han GS, Yang $\mathrm{HL}$, Tang TS, Xiaoqing C. Improvement in respiratory function after vertebroplasty and kyphoplasty. Int Orthop 2009; 33:1689-1694.

16. Wilson DC, Connolly RJ, Zhu Q, Emery JL, Kingwell SP, Kitchel S, Cripton PA, Wilson DR. An ex vivo biomechanical comparison of a novel vertebral compression fracture treatment system to kyphoplasty. Clin Biomechanics 2012; 27:346-353.

17. Olivarez LM, Dipp JM, Escamilla RF, Bajares G, Perez A, Stubbs HA, Block JE. Vertebral augmentation treatment of painful osteoporotic compression fractures with the Kiva VCF treatment system. SAS J 2011; 5:114-119. 